\title{
Capacity of the Isotropic Fading Multiple Antenna Downlink with Magnitude Feedback
}

\author{
Sudhir Srinivasa and Syed Ali Jafar \\ University of California Irvine \\ Irvine, CA 92697-2625 \\ Email: sudhirs@uci.edu,syed@ece.uci.edu
}

\begin{abstract}
We determine the capacity region of a singlecell isotropic fading multiple antenna downlink with perfect channel knowledge at the mobiles and only the knowledge of the magnitudes of the users' channel vectors at the base station. Using a scalar upperbound we are able to prove the optimality of Gaussian codebooks on this vector broadcast channel. Numerical results are provided to compare magnitude feedback against opportunistic beamforming and antenna selection.
\end{abstract}

\section{INTRODUCTION}

One of the most intriguing aspects of multiple antenna wireless systems is that the capacity benefits of using multiple antennas can depend dramatically upon the assumptions regarding the underlying time-varying channel model and how well it can be tracked at the transmitter and the receiver [1]. In practical systems, channel state information at the receiver (CSIR) is obtained from pilot symbols and blind channel estimation techniques. In the absence of delay constraints, the receiver's task is simplified somewhat because unlike the transmitter, which needs real-time channel estimates, it can wait until the end of transmission to form its channel estimate based upon the entire received sequence of coded data symbols as well as the pilot symbols. From an information theoretic perspective, the assumption of perfect CSIR allows us to deal in isolation with the issue of channel coding while ignoring the issue of designing optimal training sequences and channel estimation algorithms.

Channel state information is hard to obtain at the transmitter. In a time division duplexed system where the uplink and the downlink are using the same frequency spectrum, the reciprocity of wireless channels allows the transmitter to obtain channel state information from the received uplink transmissions. However, most current cellular systems as well as those planned in the immediate future are frequency division duplexed. For such systems, channel state information at the transmitter is obtained only by means of a feedback channel through which the receiver can send its current channel estimates to the transmitter. The task is even harder for vector broadcast channels because the number of channel coefficients that each receiver needs to estimate and instantaneously feedback is equal to the dimension of the channel vector. While, even with perfect CSIR, it is impractical for the receiver to feedback all the components of the instantaneous vector channel state to the transmitter, it may be possible to feedback partial information about the channel state. For example, the receiver may feedback only the norm (magnitude) of the instantaneous channel vector with no information about its direction. In this paper we determine the capacity region of the vector Gaussian BC with perfect CSIR and only channel magnitude information available to the transmitter. A scalar upperbound is used to prove optimality of Gaussian codebooks. We also compare magnitude feedback with other broadcast schemes such as opportunistic beamforming and antenna selection.

\section{System MODEL}

In this paper we consider the capacity region of an isotropic fading vector Gaussian BC with perfect CSIR and only channel magnitude information at the transmitter. By isotropic we mean the following: the transmitter has no knowledge of the "direction" of any user's channel vector. In other words, all directions are equivalent from the transmitter's standpoint. The precise system model is presented next.

\section{A. Broadcast Channel Model BC-V}

Consider a fading vector broadcast channel with $M$ transmit antennas at the base station and $K$ users with a single receive antenna at each user given by the input/output relationship

$$
\begin{aligned}
Y_{t}^{[1]} & =\mathbf{H}_{t}^{[1]} \mathbf{X}_{\mathbf{t}}+Z_{t}^{[1]} \\
Y_{t}^{[2]} & =\mathbf{H}_{t}^{[2]} \mathbf{X}_{\mathbf{t}}+Z_{t}^{[2]} \\
& \vdots \\
Y_{t}^{[K]} & =\mathbf{H}_{t}^{[K]} \mathbf{X}_{\mathbf{t}}+Z_{t}^{[K]}
\end{aligned}
$$

where for user $k$ at time instant $t, \mathbf{H}_{t}^{[k]}$ is the $1 \times M$ channel vector, $Y_{t}^{[k]}$ is the received scalar signal and $Z_{t}^{[k]}$ is additive white Gaussian noise (AWGN). $\mathbf{X}_{t}$ is the $M \times 1$ complex vector symbol transmitted by the base station at time instant $t$. Let the average transmit power be $P$, so that

$$
\mathrm{E}\left[\operatorname{Tr}\left(\mathbf{X X}^{\dagger}\right)\right] \leq P \text {. }
$$

Figure 1 shows the channel model for BC-V with two users. The channel fade and noise processes are ergodic and stationary. We allow the channel fade process to have memory. Thus, successive realizations of the channel and/or the noise may be correlated. For simplicity, the time index is suppressed. Isotropic fading [2] is defined as follows: 


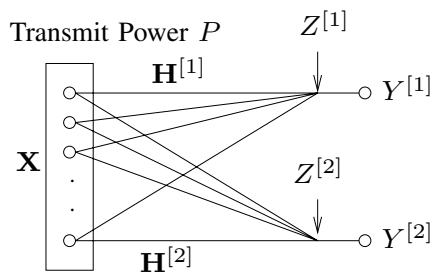

Fig. 1. BC-V

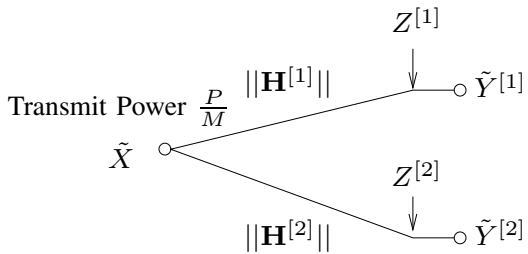

Fig. 2. BC-S
Isotropic Fading: We consider the class of channels that can be described as

$$
\mathbf{H}^{[k]}=\boldsymbol{\Phi}^{[k]} h^{[k]}
$$

where $\boldsymbol{\Phi}^{[k]}$ is a $1 \times M$ isotropically random complex unit vector and

$$
h^{[k]}=\left\|\mathbf{H}^{[k]}\right\|,
$$

the norm of the instantaneous channel vector is a non-negative scalar random variable independent of $\boldsymbol{\Phi}^{[k]}$. It is important to note that each users' channel norm may have a completely different distribution.

Recall that an isotropically random vector is one whose distribution is not affected by multiplication with a unitary matrix. It is the mathematical way to capture the notion that the vector is equally likely to point in any direction in the $M$ dimensional vector space. An example of a channel that belongs to this class is the Rayleigh fading channel with AWGN where each users' channel vector $\mathbf{H}^{[k]}$ consists of i.i.d. complex Gaussian elements $\mathbf{H}_{i}^{[k]} \sim \mathcal{N}\left(0, \alpha_{k}^{2}\right), 1 \leq i \leq M$.

Specifically, in terms of the system model BC-V, this means that $h^{[k]}$ is known to the transmitter, $1 \leq k \leq K$. Perfect CSIR is still assumed. Next we introduce the scalar upperbound.

As the name implies, the scalar upperbound is an upperbound on the capacity region of the vector broadcast channel BC-V in terms of the capacity region of a scalar broadcast channel BC-S. The new channel BC-S is described as follows:

\section{B. Broadcast Channel Model BC-S}

Associated with the broadcast channel BC-V, we define another channel model, BC-S, with input/output relationship

$$
\begin{aligned}
\tilde{Y}^{[1]} & =h^{[1]} \tilde{X}+Z^{[1]} \\
\tilde{Y}^{[2]} & =h^{[2]} \tilde{X}+Z^{[2]} \\
& \vdots \\
\tilde{Y}^{[K]} & =h^{[K]} \tilde{X}+Z^{[K]}
\end{aligned}
$$

Notice that the input $\tilde{X}$ is a scalar, and each users' channel is also a scalar equal to the norm of the corresponding original vector channel. The additive noise experienced by each user is the same as in the vector broadcast channel model BC-V. The new transmit power constraint is

$$
\mathrm{E}\left[|\tilde{X}|^{2}\right] \leq \frac{P}{M} .
$$

Figure 2 shows the channel model for BC-S with two users. Of course, an alternate representation of the BC-S channel is where the transmit power constraint is $P$ instead of $\frac{P}{M}$ and the users' channels are $h^{[k]} / \sqrt{M}$ instead of $h^{[k]}$. To avoid confusion, in this paper we will use the system model as represented in Figure 2.

The following theorem establishes the scalar upperbound for the vector broadcast channel with magnitude feedback.

Theorem 1: The capacity region of the vector fading broadcast channel BC-V with perfect CSIR and magnitude feedback is contained within the capacity region of the scalar fading broadcast channel BC-S with perfect CSIR and perfect CSIT.

Proof of Theorem 1: The proof for the applicability of the scalar upperbound is identical to that for Theorem 1 of [2] if we also assume magnitude feedback in the scalar fading broadcast system model BC-S. Thus, the capacity region of the vector broadcast channel BC-V with magnitude feedback is contained within the capacity region of the scalar broadcast channel BC-S with magnitude feedback. Interestingly, magnitude feedback for the scalar broadcast channel constitutes the entire channel state information. Thus, we can equivalently state that the capacity region of the vector broadcast channel BC-V with perfect CSIR and magnitude feedback is contained within the capacity region of the scalar broadcast channel with perfect CSIR and perfect CSIT.

For our second result in this section we need the assumption that the additive noise is white and Gaussian. Under this assumption, the capacity region of the scalar fading broadcast channel with perfect CSIR and CSIT has already been found by $\mathrm{Li}$ and Goldsmith (Theorem 3.1 of [3]). From $\mathrm{Li}$ and Goldsmith's result we can directly state the following:

Theorem 2: The capacity region of the scalar fading Gaussian broadcast channel BC-S with perfect CSIR and perfect CSIT is

$$
C(P)=\cup_{\mathcal{P} \in \mathcal{F}} C_{C D}(\mathcal{P}),
$$

where

$$
\begin{aligned}
& C_{C D}(\mathcal{P})=\{ R_{k} \leq \mathrm{E}_{\vec{h}^{[\cdot]}}[\log (1+ \\
&\left.\left.\frac{\left(h^{[k]}\right)^{2} P_{k}\left(\vec{h}^{[\cdot]}\right)}{1+\sum_{j=1}^{K}\left(h^{[j]}\right)^{2} P_{j}\left(\vec{h}^{[\cdot]}\right) 1\left(h^{[j]}>h^{[k]}\right)}\right)\right] \\
&1 \leq k \leq K\}
\end{aligned}
$$

is the achievable region with superposition coding and successive decoding with power allocation policy $\mathcal{P}$. The joint channel state of all users is denoted by the vector $\vec{h}^{[\cdot]}=$ $\left\{h^{[1]}, h^{[2]}, \cdots, h^{[K]}\right\} . P_{k}\left(\vec{h}^{[\cdot]}\right)$ is the transmit power allocated to user $k$ under power policy $\mathcal{P}$. $\mathcal{F}$ is the set of all permissible power policies that satisfy the average power constraint 
$\sum_{k=1}^{K} P_{k}\left(\vec{h}^{[\cdot]}\right) .1(\cdot)$ denotes the indicator function $(1(x)=1$ if $x$ is true and 0 otherwise). Note that the way this theorem is stated it assumes that no two users have the same channel magnitude. As mentioned in [3] the case when, for example users $i$ and $j$ have identical channel magnitudes can easily be handled by viewing the two users as one user, applying superposition coding and successive decoding to the resulting $K-1$ users. Time sharing is used for users $i$ and $j$. Thus, for simplicity, we will assume henceforth in this section that all the users have distinct vector channel magnitudes.

Using Li and Goldsmith's result stated above we prove the following Theorem:

Theorem 3: The capacity region of the isotropic vector fading Gaussian broadcast channel BC-V with perfect CSIR and only a knowledge of $\vec{h}^{[\cdot]}$ at the transmitter (magnitude feedback) is identical to the capacity region of the scalar fading Gaussian broadcast channel BC-S described in Theorem 2. Note that this theorem does not make any assumption regarding the distribution of users' magnitudes.

Proof of Theorem 3: In order to prove Theorem 3 we only need to prove achievability. The converse is already proved from the scalar upperbound. For achievability, we start by considering a fixed channel magnitude vector $\vec{h}^{[\cdot]}$. Without loss of generality suppose the users' fixed magnitudes are ordered as

$$
h^{[1]}<h^{[2]}<\cdots<h^{[K]} .
$$

Note that conditioned on the given $h^{[k]}$, each user's channel is just an isotropic random vector of the corresponding magnitude. Also, since the broadcast channel capacity depends only on the marginal distributions $p\left(Y^{[i]} \mid \mathbf{X}\right.$ ) (see [4]), we can assume that all the users' channel vectors are parallel. The common direction of all the channels is known to each receiver (perfect CSIR) but not known to the transmitter.

Lemma 1: The vector fading Gaussian broadcast channel BC-V with a fixed channel magnitude vector $\vec{h}^{[\cdot]}$ is stochastically degraded in the same order as the users' channel magnitudes.

Proof of Lemma 1: In fact for fixed channel magnitudes, the vector fading Gaussian broadcast channel BC-V can be transformed into a physically degraded broadcast channel without affecting its capacity. Suppose each user scales its received signal by the reciprocal of its channel magnitude $h^{[k]}$. Then, all the users have identical channels but different noise variances. The $k^{t h}$ user's additive noise is white Gaussian with variance $\frac{1}{\left(h^{[k]}\right)^{2}}$. Now, without affecting the marginals [4] we can assume

$$
\begin{array}{r}
Z^{[i]}=Z^{[i+1]}+\left(\sqrt{\left(\frac{1}{h^{[i]}}\right)^{2}-\left(\frac{1}{h^{[i+1]}}\right)^{2}}\right) N_{i} \\
i=\{1,2, \cdots, K-1\}
\end{array}
$$

$N_{i}$ are i.i.d. zero mean unit variance circularly symmetric complex Gaussian random variables. Each user's received signal is the same as the next user's received signal with some independent noise added to it. Thus, the users are physically degraded. If we do not assume (10) then the users are only stochastically degraded.

Lemma 2: For the vector fading Gaussian broadcast channel BC-V with a fixed channel magnitude vector $\vec{h}^{[\cdot]}$ and a fixed power allocation vector $\mathcal{P}=$ $\left\{P_{1}\left(\vec{h}^{[\cdot]}\right), P_{2}\left(\vec{h}^{[\cdot]}\right), \cdots, P_{K}\left(\vec{h}^{[\cdot]}\right)\right\}$ the following rate tuples are "achievable" with superposition coding and successive decoding

$$
\begin{aligned}
& \left\{\left(R^{[1]}, R^{[2]}, \cdots, R^{[K]}\right):\right. \\
& R^{[k]} \leq \log \left(1+\frac{h^{[k]} P_{k}\left(\vec{h}^{[\cdot]}\right)}{1+\sum_{j=1}^{K} h^{[j]} P_{j}\left(\vec{h}^{[\cdot]}\right) 1\left(h^{[j]}>h^{[k]}\right)}\right) \\
& 1 \leq k \leq K\}
\end{aligned}
$$

By "achievable" we mean the rate tuple lies within the capacity region.

Proof of Lemma 2: For a stochastically degraded channel the capacity region is given by

$$
\begin{aligned}
C=\quad & \cup_{p\left(\mathbf{U}_{\mathbf{1}}, \cdots, \mathbf{U}_{\mathbf{K}-\mathbf{1}}, \mathbf{X}\right): \mathbf{U}_{\mathbf{1}} \rightarrow \cdots \rightarrow \mathbf{U}_{\mathbf{K}-\mathbf{1}} \rightarrow \mathbf{X} \rightarrow\left(\mathbf{Y}^{[1]}, \cdots, \mathbf{Y}^{[\mathbf{K}]}\right)} \\
& \left\{\left(R^{[1]}, \cdots, R^{[K]}\right) \in \mathcal{R}_{+}^{K}:\right. \\
& R^{[1]} \leq I\left(\mathbf{U}_{\mathbf{1}} ; Y^{[1]}\right) \\
& R^{[k]} \leq I\left(\mathbf{U}_{\mathbf{k}} ; Y^{[k]} \mid \mathbf{U}_{\mathbf{1}}, \cdots, \mathbf{U}_{\mathbf{k}-\mathbf{1}}\right) \\
& k \in\{2, \cdots, K-1\} \\
& \left.R^{[K]} \leq I\left(\mathbf{X} ; Y^{[K]} \mid \mathbf{U}_{\mathbf{1}}, \cdots, \mathbf{U}_{\mathbf{K}-\mathbf{1}}\right)\right\}
\end{aligned}
$$

To obtain an achievable point within the capacity region let us substitute $\left(\mathbf{U}_{\mathbf{1}}, \mathbf{U}_{\mathbf{2}}, \cdots, \mathbf{U}_{\mathbf{K}}, \mathbf{X}\right)$ jointly Gaussian into (12) such that:

$$
\begin{aligned}
\mathbf{X} & =\mathbf{U}_{\mathbf{1}}+\mathbf{U}_{\mathbf{2}}+\cdots+\mathbf{U}_{\mathbf{K}} \\
\mathbf{U}_{\mathbf{k}} & \sim \mathcal{N}\left(0, \frac{P_{k}\left(\vec{h}^{[\cdot]}\right)}{M} I_{M}\right)
\end{aligned}
$$

and $\mathbf{U}_{1}, \mathbf{U}_{2}, \cdots, \mathbf{U}_{\mathbf{K}}$ are independent. Then it is easily seen that the resulting achievable rate region is the same as (11). This completes the proof of Lemma 2.

Finally, to prove Theorem 3 we use the idea of multiple codebooks, such that each codebook corresponds to a joint channel magnitude vector $\vec{h}^{[\cdot]}$. Since both the transmitter and receiver know the instantaneous channel magnitudes it is possible to multiplex these codebooks such that for every channel realization the corresponding codebook is used. Each codebook is designed for a rate that can be supported by the channel if the channel magnitudes are constants represented by the channel magnitude vector $\vec{h}^{[\cdot]}$ corresponding to that codebook. The overall rate vector achieved in this manner is the average of the rate vectors of all the codebooks, where the averaging is over the distribution of the joint channel magnitudes vector $\vec{h}^{[\cdot]}$. Since this argument is quite standard we will simply refer the reader to [5] and [6]. It is also shown in [6] that multiple codebooks are not necessary to achieve this capacity region. 


\section{Opportunistic BeAmForming, Antenna SELECTION AND MAGNitude FEEDBACK}

In this section, we qualitatively describe two other possible transmission schemes the base station can adopt - opportunistic beamforming and antenna selection.

Opportunistic beamforming [7] uses multiple antennas at the base station to send the same signal from each of the $M$ antennas multiplied by a gain $\left(\sqrt{\beta_{m}(t)}\right)$ and a phase shift $\left(e^{j \cdot \theta_{m}(t)}\right)$ at the $m^{t h}$ antenna. These gains and phases of the antennas are varied in a pseudo random manner with the constraint that $\sum_{m=1}^{M} \beta_{m}(t)=1$. The basic idea in opportunistic beamforming is that in a heavily populated system, there is always at least one user whose beamforming configuration is very close to the transmitted vector. At any time, the throughput can be maximized by allocating resources to the best user at that time.

In transmit antenna selection, the base station has a fixed number of antennas. Each user determines the best subset of antennas and sends this information to the transmitter. The transmission is then done using only the subset of antennas chosen by that user. We will only look at single antenna selection for future comparisons, where the user selects the best transmit antenna at the base station. The transmitter then puts all the available power into that single antenna during transmission. Single antenna selection is a special case of quantized beamforming, where the number of transmit beamforming vectors is equal to the number of transmit antennas.

\section{NumERiCAL RESUlts}

In this section, we present numerical results obtained with magnitude feedback, and compare the magnitude feedback scheme with opportunistic beamforming and single antenna selection. For each of the three schemes discussed, we consider four simulation scenarios:

- I.I.D and Non I.I.D Rayleigh fading: The I.I.D case has users with identically distributed Rayleigh fading channels with an average receive SNR of $5.965 \mathrm{~dB}$. In the non I.I.D case, the channel for each user is Rayleigh, but the average SNR is generated independently for each of the $K$ users according to the CDF given in [8].

- I.I.D and Non I.I.D Rician fading: The I.I.D case has identically distributed Rician fading channels with $\kappa=$ 5 and an average SNR of 5.965dB. $\kappa$ is the K-factor of the distribution, defined as $E_{\text {direct }} / E_{\text {specular }}$, where $E_{\text {direct }}$ and $E_{\text {specular }}$ are the energies in the direct and specular paths respectively. For the non I.I.D part, the channel for each user is Rician with $\kappa=5$, and the average SNR is generated independently for each of the $K$ users according to the CDF given in [8].

The performance of the three schemes for each of the four channel conditions above is simulated with and without scheduling of users in the system. We use the proportional fair (PF) scheduling algorithm described in [7], with $t_{c}=400$ time slots in all our simulations.

\section{A. Performance with increase in number of users}

In Fig 3, average throughputs for magnitude feedback, opportunistic beamforming and antenna selection are plotted against the number of users $(K)$ in the system. There is no scheduling of users. It can be seen that for small number of users in the system, magnitude feedback achieves higher throughputs than opportunistic beamforming. This is manifested prominently in the unscheduled non I.I.D Rayleigh and Rician cases. As the number of users increases, the probability of a user being in its beamforming configuration is higher. As a result, opportunistic beamforming performs better when the system has a large number of users.
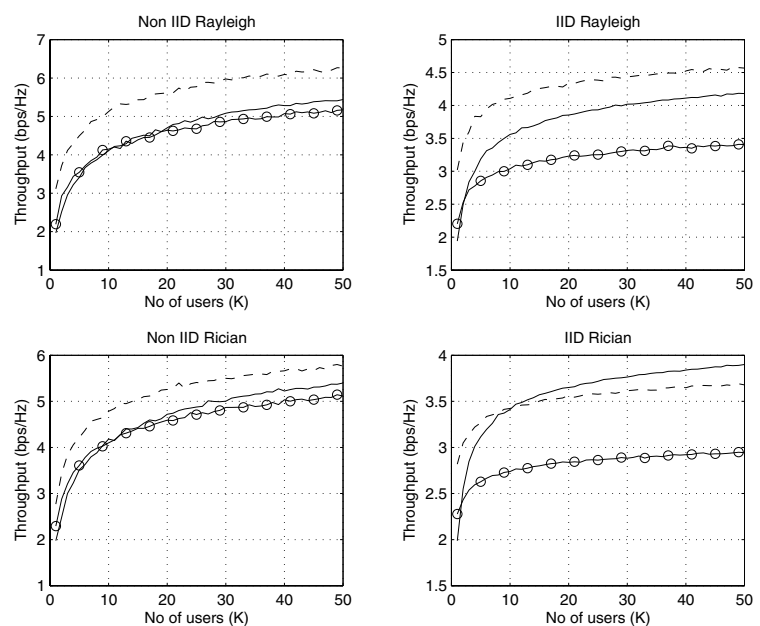

Fig. 3. Average throughput (in bps/Hz) of opportunistic beamforming (solid lines), magnitude feedback (solid lines marked with circles) and single antenna selection (dashed lines) against the number of users with $M=4$ base station antennas. The users are not scheduled.

In Figure 4, the three schemes are compared with the users time shared using the PF scheduling algorithm. The plots follow the same trend as for the unscheduled case.

\section{B. Performance with increase in number of base station antennas}

We now compare the three schemes considered with respect to the number of transmit antennas $(M)$ in the system. We show the average throughputs for the three schemes with $K=$ 5 and $K=50$ users without scheduling in Figure 5. Figure 6 shows the throughputs for the three schemes when the users are scheduled according to the PF scheduling algorithm.

We observe that the throughputs for magnitude feedback decrease with an increase in the number of transmit antennas. This may seem counter-intuitive, as one would expect that if that is the case, then the base station would use only a subset of the available antennas for transmission. Thus, the sum-capacity, even if it does not increase with more antennas, should not decrease as the number of transmit antennas is increased. However, it is important to note that under the magnitude feedback model, the transmitter only knows the magnitude of the channel vector comprising of all the transmit antenna elements. At any time $t$, the base station has 

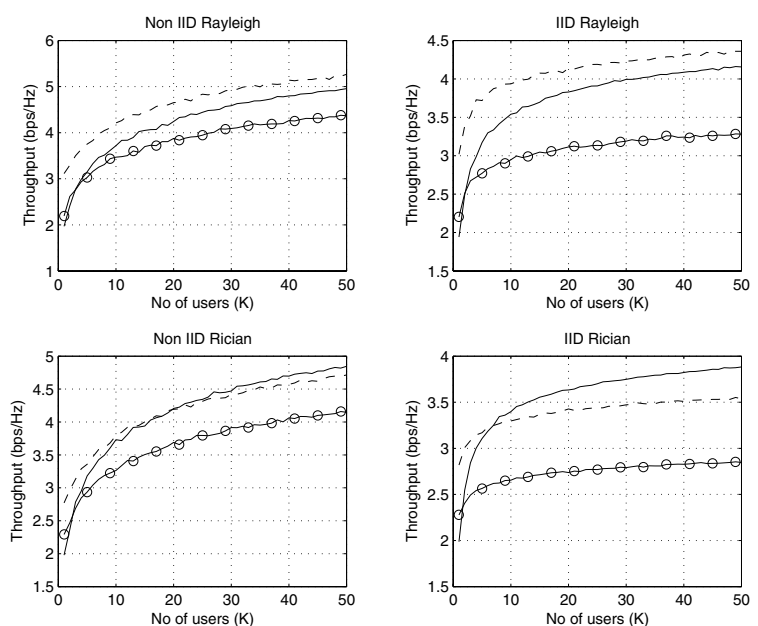

Fig. 4. Performance of opportunistic beamforming (solid lines), magnitude feedback (solid lines marked with circles) and single antenna selection (dashed lines) with PF scheduling against the number of users. $M=4$ base station antennas is assumed.

knowledge of $h^{[k]}$ for the $k^{t h}$ user and this does not provide magnitude information for any sub-vector corresponding to a smaller subset of antennas. Given the magnitude feedback for the isotropic channel vector corresponding to $M$ antennas it is therefore optimal to transmit on all $M$ antennas and the total throughput can be smaller as the number of transmit antennas increases, as indicated by the plots.

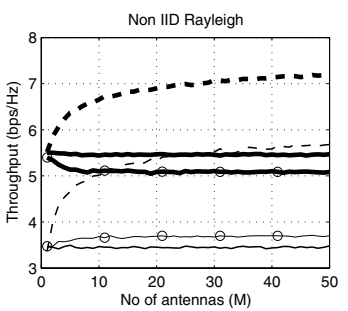

Non IID Rician

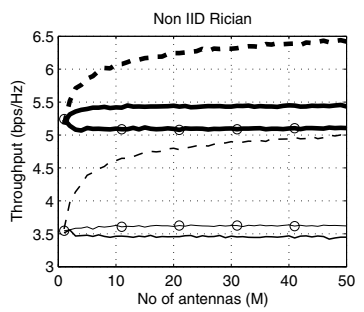

Fig. 5. Performance of opportunistic beamforming (solid lines), magnitude feedback (solid lines marked with circles) and single antenna selection (dashed lines) with respect to number of base station antennas. Thin lines denote plots for $K=5$ users, whereas thick lines represent $K=50$ users. The base station does not schedule the users.

\section{Conclusions}

We determine the full capacity region of an isotropic fading vector broadcast channel when the channel state is perfectly known at the receivers and only the magnitudes of the channel vectors are available at the transmitter. Using a scalar
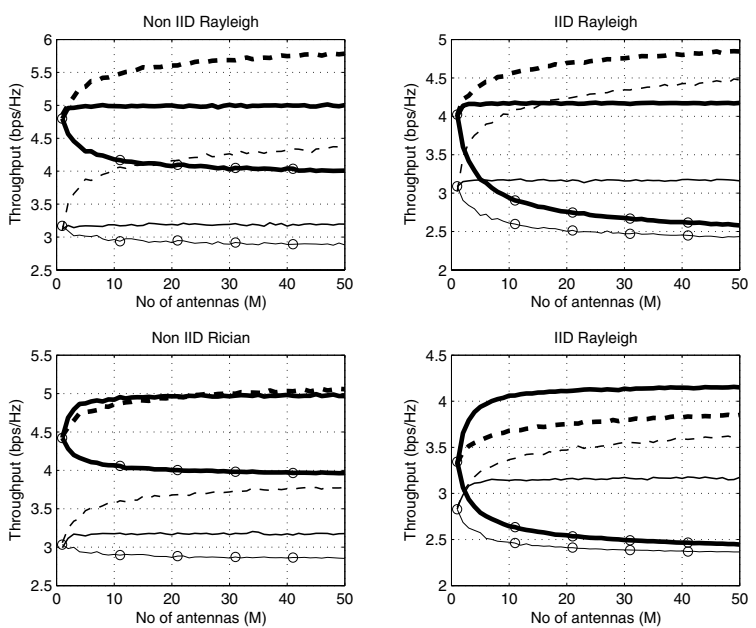

Fig. 6. Average throughput (in $\mathrm{bps} / \mathrm{Hz}$ ) of opportunistic beamforming (solid lines), magnitude feedback (solid lines marked with circles) and single antenna selection (dashed lines) with PF scheduling against the number of base station antennas. Thin lines denote plots for $K=5$ users, whereas thick lines represent $K=50$ users.

upperbound we are able to prove the optimality of Gaussian codebooks on this vector broadcast channel.

From the results obtained, we see that for a small number of users in the system, magnitude feedback outperforms opportunistic beamforming. However, increasing the number of transmit antennas decreases the throughput in the magnitude feedback case. This behavior stems from the fact that the base station cannot determine the channel magnitude for a subset of antennas used from the overall magnitude fed back from each user. On the other hand, the lack of directional information at the transmitter is responsible for the small throughputs achieved by magnitude feedback. This indicates that at least some directional information about the users is also required to effectively employ multiple antennas at the base station.

\section{REFERENCES}

[1] A. Goldsmith, S. A. Jafar, N. Jindal, and S. Vishwanath, "Capacity limits of MIMO channels," IEEE Journal on Selected Areas in Communications, vol. 21, pp. 684-702, June. 2003.

[2] S. Jafar and A. Goldsmith, "On the capacity region of the vector fading broadcast channel with no CSIT," in Proceedings of IEEE ICC 2004, June 2004.

[3] L. Li and A. Goldsmith, "Capacity and optimal resource allocation for fading broadcast channels-Part I: Ergodic capacity," IEEE Trans. Inform. Theory, vol. 47, pp. 1083-1102, March 2001.

[4] T. M. Cover, "Broadcast channels," IEEE Trans. Inform. Theory, vol. 18, pp. 2-14, Jan. 1972.

[5] A. Goldsmith and P. Varaiya, "Capacity of fading channels with channel side information," IEEE Trans. Inform. Theory, vol. 43, pp. 1986-1992, Nov. 1997.

[6] G. Caire and S. Shamai, "On the capacity of some channels with channel state information," IEEE Trans. Inform. Theory, vol. 45, pp. 2007-2019, Sept 1999.

[7] P. Viswanath, D. Tse and R. Laroia, "Opportunistic Beamforming Using Dumb Antennas," IEEE Trans. Inform. Theory, vol. 48, pp. 1277-1294, June 2002.

[8] H. Viswanathan, S. Venkatesan and H. Huang, "Downlink capacity evaluation of Cellular Networks with Known Interference Cancellation" IEEE Journal on Selected Areas in Communications, vol. 21, no. 5, pp. 802-811, Jun 2003. 